\title{
Utility of a Serum Aspergillus Galactomannan Assay in Diagnosis of Invasive Pulmonary Aspergillosis in HIV/AIDS Patients: A Prospective Analysis
}

\author{
Ravinder Kaur ${ }^{1 *}$, Bhanu Mehra ${ }^{2}$, Megh Singh Dhakad ${ }^{3}$, Ritu Goyal ${ }^{3}$, Preena Bhalla ${ }^{3}$ and Richa Dewan ${ }^{4}$ \\ ${ }^{1}$ Department of Microbiology, Lady Hardinge Medical College and Associated Kalawati Saran Children Hospital, New Delhi, India \\ ${ }^{2}$ Department of Microbiology, Dr. Baba Saheb Ambedkar Medical College and Hospital, New Delhi, India \\ ${ }^{3}$ Department of Microbiology, Maulana Azad Medical College and Lok Nayak Hospital, New Delhi, India \\ ${ }^{4}$ Department of Medicine, Maulana Azad Medical College and Lok Nayak Hospital, New Delhi, India
}

\begin{abstract}
Background: Invasive pulmonary aspergillosis (IPA) is a life threatening infection among immunocompromised patients. A timely and precise diagnosis of this condition is often precluded by lack of sensitive and specific noninvasive diagnostic tools. Detection of galactomannan has emerged as a promising tool in this regard. While the analytical performance of serum galactomannan assay has been extensively evaluated in various high risk groups, its possible utility in HIV/AIDS patients is yet to be explored. The present study was undertaken to evaluate the yield of a serum galactomannan assay for diagnosis of IPA in a cohort of HIV/AIDS patients.

Methods: 49 febrile HIV reactive patients with co-existent symptomatic AIDS were prospectively enrolled for the evaluation. Blood samples were drawn from each patient and serum galactomannan screening was performed employing the commercially available serum galactomannan assay, Platelia ${ }^{\mathrm{TM}}$ Aspergillus EIA. An optical density index $>0.5$ for at least two serum samples was considered the defining criteria for a positive result. Furthermore, sputum samples were collected and direct microscopic examination ( $10 \%$ potassium hydroxide wet mount) and fungal cultures were performed.

Result: In accordance with the definitions laid down under EORTC/MSG (European Organization for Research and Treatment of Cancer / Mycoses Study Group) criteria, ten cases of probable and 23 cases of possible IPA were diagnosed. The sensitivity, specificity, and positive and negative predictive values of Platelia ${ }^{\text {TM }}$ Aspergillus EIA for patients with probable IPA were 90\%, 100\%, 100\% and 94.12 \% respectively.

Conclusion: Serum galactomannan assay has a high sensitivity and specificity and when complemented with relevant lower respiratory tract symptomatology and imaging findings, can serve as a useful adjunct to conventional modalities for the diagnosis of IPA in HIV/AIDS patients.
\end{abstract}

Keywords: Galactomannan, HIV/AIDS, Invasive Pulmonary Aspergillosis, Serum

\section{Introduction}

Invasive mycoses such as invasive pulmonary aspergillosis (IPA) are a significant cause of mortality and morbidity in the immunosuppressed population. ${ }^{[1,2]}$ In human immunodeficiency virus (HIV) infected persons in particular, the median survival is nearly 3 months following a diagnosis of IPA. ${ }^{[3]}$

An accurate diagnosis of IPA is a challenge till date. The clinical signs and symptoms are generally non-specific and chest roentgenographic features are frequently absent. $[4,5]$ High resolution computed tomography (HRCT) findings are also not independently diagnostic and fail to differentiate IPA from candidiasis, cryptococcosis and bacterial infections. $[6,7,8]$ A histopathological demonstration of invasion of lung tissue with fungal hyphae, in conjunction with isolation of Aspergillus species from tissue samples is generally considered the reference standard for a diagnosis. ${ }^{[9,10]}$ However, the diagnostic yield of routine mycological procedures such as microscopy and culture is generally low. ${ }^{[8,11]}$ Moreover, interpretation is often complicated by difficulty in discriminating invasive disease from colonization. ${ }^{[11]}$ Tissue biopsy, too, is invasive and seldom not feasible due to serious underlying condition of the patients and frequent presence of hypoxia, cytopenias, coagulation abnormalities and hemodynamic disturbances. ${ }^{[4,8,12]}$

Keeping in view the limitations of the currently available diagnostic modalities and with the understanding that early diagnosis of IPA would result in better patient outcomes, the significance of newer and rapid diagnostic platforms 
in guiding prompt therapeutic management cannot be undermined. This has led to a search for non-invasive and non-culture- based approaches for an early and rapid diagnosis of invasive aspergillosis. Advent of biomarker assays has been a major breakthrough in this regard. These tests detect circulating Aspergillus antigens, metabolites or nucleic acid targets as surrogate markers for diagnosing this invasive condition. ${ }^{[13]}$ While molecular methods such as polymerase chain reaction have been evaluated for this purpose, lack of standardization and validation of these procedures has limited their diagnostic utility. ${ }^{[14]}$

The second approach, that is, detection of cell wall components such as galactomannan and 1, 3- $\beta$-D-glucan offers a promising alternative. Galactomannan, a cell wall component, is not released into the circulation till the fungus invades the endothelial compartment and is thus a marker of angioinvasion by the fungus. ${ }^{[15]}$ Initial assays for detection of this antigen included latex agglutination test and suffered from low sensitivity. ${ }^{[4,5]}$ The currently available technique is a double sandwich enzyme linked immunosorbent assay (ELISA), that has been extensively evaluated and has also been approved by the United States Food and Drug Administration for diagnostic use. ${ }^{[4,16]}$

While assays for galactomannan detection are widely available in India and have been used in high risk population groups such as stem cell transplant recipients and patients with hematological malignancies, there is virtually no published data regarding its possible use for diagnosis of IPA in HIV positive patients. ${ }^{[17,18]}$ The present study was undertaken to detect circulating serum galactomannan antigen in a group of febrile HIV reactive patients with co-existent symptomatic acquired immunodeficiency syndrome (AIDS) and to assess its yield and diagnostic performance for diagnosis of IPA.

\section{Materials and Methods}

The present, cross-sectional, single institutional and noninterventional study was conducted in a cohort of HIV reactive patients seeking medical care at a tertiary care teaching hospital in New Delhi, India. From October 2008 to September 2011, HIV positive patients from various inpatients units as well as those attending anti-retroviral treatment clinic were prospectively analyzed. Patients were eligible for enrollment if they had a body temperature $>38^{\circ} \mathrm{C}$ with co-existent symptomatic AIDS (a host factor criterion consistent with invasive pulmonary aspergillosis). ${ }^{[10]}$ A convenient sample of 234 consecutive HIV positive subjects was chosen from which 49 subjects fulfilling the inclusion criteria were selected. HIV reactive patients on long term steroids or other immunosuppressive agents; those receiving piperacillin-tazobactam or amoxycillin- clavulanate; and those with a concurrent neutropenia were excluded from the analysis. The study protocol was approved by the institutional ethics committee and written informed consent was requested from all enrolled subjects before recruiting them for the study.

A standardized, pre-designed, structured proforma was used to collect information regarding patients' sociodemographic characteristics, clinical profile, radiological findings and various laboratory parameters. All the patient data were anonymized in order to maintain confidentiality.

Blood samples were collected following standard precautions and transported to the laboratory. Sera were separated and stored at $-70{ }^{\circ} \mathrm{C}$ till the serum galactomannan assay was performed. All patients were screened for serum galactomannan employing the Platelia $^{\mathrm{TM}}$ Aspergillus EIA (Bio- Rad Laboratories). The test was performed and analyzed in accordance with the procedural and interpretative details as mentioned in the manufacturer's guidelines. An optical density index (ODI) $>0.5$ was considered as positive. While a patient with a negative result with Platelia ${ }^{\mathrm{TM}}$ Aspergillus EIA was considered as a galactomannan negative case, a repeat sample was tested in patients with a positive result. The test result was considered positive only when the second sample yielded the same result. Furthermore, 20 healthy HIV negative individuals with no clinical, radiological or microbiological evidence of invasive pulmonary aspergillosis were recruited as controls to establish the validity of the Platelia ${ }^{\mathrm{TM}}$ Aspergillus EIA kit.

In addition, sputum as a respiratory source for mycological assessment was collected from each patient and subjected to direct microscopic examination $(10 \%$ potassium hydroxide wet mount) and fungal culture on Sabouraud's dextrose agar. The cultures were examined every second day for up to six weeks before they were reported as sterile. Any suspected fungal growth during this period was examined for gross colony morphology including color, texture, topography and the underside or reverse. Further confirmation of the isolates was done by slide culture technique, preparing lactophenol cotton blue mount and examining with the aid of $10 \mathrm{X}$ and $40 \mathrm{X}$ objective of the microscope. Speciation of Aspergillus isolates was done based on characteristic morphology of their hyphae and spores under microscopy and employing tests as per standard protocol. ${ }^{[19]}$ Any positive result on microscopy and/or culture was confirmed by subsequent sampling and repeat demonstration/isolation.

Each patient was classified as probable, possible and nonIPA as per definitions laid down under EORTC/MSG (European Organization for Research and Treatment of 
Cancer / Mycoses Study Group) criteria. ${ }^{[10]}$ While probable cases required a compulsory microbiological proof in addition to host factors and clinical criteria, possible cases required compatible host factors in conjunction with either clinical features or mycological findings.

Descriptive statistics were performed with arithmetic mean and standard deviation calculated for central tendencies and median for non-normal/skewed distributions. The analysis was performed using the Epi info software, version 3.5.3, CDC, Atlanta, GA, USA. In addition, serum galactomannan assay and lower respiratory tract Aspergillus isolation were assessed with respect to sensitivity, specificity, positive predictive value, and negative predictive value of these tests. The values were calculated with their respective confidence interval of 95\%. For performing the calculations, possible IPA cases were not included in the analysis.

\section{Results}

Thirty three $(67.3 \%)$ of our study participants were drawn from the antiretroviral treatment clinic while 16 (32.7\%) were recruited from the various inpatient units. The mean and median age of the study subjects was $35.24 \pm 9.43$ years and 34 years respectively (range $=18-61$ years). The study population comprised of $36(73.5 \%)$ males and $13(26.5 \%)$ females with a male to female ratio of $2.8: 1$. The study group included $42(85.7 \%)$ married and seven (14.3\%) unmarried participants. Heterosexual promiscuous was the most commonly reported mode of HIV transmission documented in $43(87.8 \%)$ study subjects followed by intravenous drug abuse in four $(8.1 \%)$ and blood transfusion in two (4.1\%). Fever, cough and dyspnea were the most common clinical manifestations noted in 49 (100\%), $32(65.3 \%)$ and $15(30.6 \%)$ patients respectively. A high proportion of patients $(29 ; 59.2 \%)$ had tuberculosis as an underlying predisposing condition and ten (20.4\%) patients had a Pneumocystis carinii pneumonia. The mean and median CD4 count of the study population was $196.57 \pm 109.62$ cells $/ \mu 1$ and 178 cells $/ \mu 1$ respectively with a range of $16-423$ cells $/ \mu 1$. In addition, while a normal chest radiograph was documented in 25 (51\%) patients, the remaining had radiographic abnormalities as follows: pleural effusion in $15(30.6 \%)$; infiltrates in five (10.2\%); opacity in three $(6.1 \%)$ and hyperinflation in one $(2 \%)$.

Employing the EORTC/MSG criteria, ten cases of probable and 23 cases of possible invasive pulmonary aspergillosis were diagnosed. The characteristics of HIV reactive patients diagnosed with a probable IPA are summarized in Table 1 . Chest radiography of eight $(80 \%)$ of these patients revealed a pleural effusion, while infiltrates were seen in two $(20 \%)$. HRCT scan revealed the characteristic aircrescent sign in only one of these patients. Furthermore, CT confirmed the presence of pleural effusion in the eight cases and documented the presence of new infiltrates not fulfilling major criteria in another patient.

The diagnostic yield of sputum culture, direct microscopy and Platelia ${ }^{\mathrm{TM}}$ Aspergillus EIA in HIV reactive patients with probable IPA was 30\% (3/10), 30\% (3/10) and 90\% (9/10) respectively. The sensitivity, specificity and positive and negative predictive values of Platelia ${ }^{\mathrm{TM}}$ Aspergillus EIA were 90\% (55.50-99.75\%), 100\% (79.41- 100\%), 100\% (66.37- 100\%) and 94.12\% (71.31- 99.85\%) respectively, while the corresponding performance characteristics of lower respiratory tract Aspergillus isolation were 30\% (6.67- 65.25\%), 100\% (79.41- 100\%), 100\% (29.24$100 \%)$ and $69.57 \%(47.08-86.79 \%)$ respectively.

Further, it is noteworthy to know that two healthy HIV negative controls with no signs and symptoms of invasive pulmonary aspergillosis gave positive results with Platelia $^{\mathrm{TM}}$ Aspergillus EIA, but only on one occasion. A repeat test performed on a fresh blood specimen drawn from these patients did not yield a positive result, and thus these could not be considered as confirmed false positives.

Table 1: Characteristics of HIV*/ AIDS $\dagger$ patients with probable IPA $(n=10)$

\begin{tabular}{|c|c|c|c|c|c|c|c|c|c|c|}
\hline $\begin{array}{l}\text { S. } \\
\text { No. }\end{array}$ & Age & Sex & $\begin{array}{c}\text { Clinical } \\
\text { presentation }\end{array}$ & $\begin{array}{l}\text { Mode of HIV } \\
\text { transmission }\end{array}$ & $\begin{array}{c}\text { Underlying } \\
\text { lung } \\
\text { disease }\end{array}$ & $\begin{array}{l}\text { CD4 } \\
\text { count }\end{array}$ & $\begin{array}{c}\text { Direct } \\
\text { microscopy }\end{array}$ & $\begin{array}{l}\text { Fungal } \\
\text { culture }\end{array}$ & $\begin{array}{l}\text { Aspergillus } \\
\text { species } \\
\text { isolated }\end{array}$ & $\begin{array}{c}\text { Aspergillus } \\
\text { galactomannan } \\
\text { antigen }\end{array}$ \\
\hline 1. & 28 & $\mathrm{M} \S$ & Fever, cough & $\begin{array}{l}\text { Heterosexual } \\
\text { contact }\end{array}$ & TB\| & 107 & $\begin{array}{l}\text { Fungal } \\
\text { hyphae } \\
\text { seen }\end{array}$ & Positive & $\begin{array}{l}\text { Aspergillus } \\
\text { flavus }\end{array}$ & Negative \\
\hline 2. & 52 & M & $\begin{array}{c}\text { Fever, cough, } \\
\text { dyspnea }\end{array}$ & $\begin{array}{c}\text { Heterosexual } \\
\text { contact }\end{array}$ & - & 85 & $\begin{array}{c}\text { Fungal } \\
\text { hyphae not } \\
\text { seen }\end{array}$ & Negative & - & Positive \\
\hline 3. & 49 & $M$ & $\begin{array}{c}\text { Fever, } \\
\text { dyspnea }\end{array}$ & $\begin{array}{l}\text { Heterosexual } \\
\text { contact }\end{array}$ & TB & 168 & $\begin{array}{l}\text { Fungal } \\
\text { hyphae not } \\
\text { seen }\end{array}$ & Negative & - & Positive \\
\hline
\end{tabular}




\begin{tabular}{|c|c|c|c|c|c|c|c|c|c|c|}
\hline $\begin{array}{l}\text { S. } \\
\text { No. }\end{array}$ & Age & Sex & $\begin{array}{c}\text { Clinical } \\
\text { presentation }\end{array}$ & $\begin{array}{l}\text { Mode of HIV } \\
\text { transmission }\end{array}$ & $\begin{array}{l}\text { Underlying } \\
\text { lung } \\
\text { disease }\end{array}$ & $\begin{array}{l}\text { CD4 } \\
\text { count }\end{array}$ & $\begin{array}{c}\text { Direct } \\
\text { microscopy }\end{array}$ & $\begin{array}{l}\text { Fungal } \\
\text { culture }\end{array}$ & $\begin{array}{l}\text { Aspergillus } \\
\text { species } \\
\text { isolated }\end{array}$ & $\begin{array}{c}\text { Aspergillus } \\
\text { galactomannan } \\
\text { antigen }\end{array}$ \\
\hline 4. & 38 & M & $\begin{array}{c}\text { Fever, } \\
\text { dyspnea }\end{array}$ & $\begin{array}{l}\text { Heterosexual } \\
\text { contact }\end{array}$ & TB & 85 & $\begin{array}{c}\text { Fungal } \\
\text { hyphae not } \\
\text { seen }\end{array}$ & Negative & - & Positive \\
\hline 5. & 42 & $M$ & $\begin{array}{l}\text { Fever, } \\
\text { dyspnea }\end{array}$ & $\begin{array}{l}\text { Heterosexual } \\
\text { contact }\end{array}$ & $\mathrm{TB}, \mathrm{PCP}^{* *}$ & 54 & $\begin{array}{l}\text { Fungal } \\
\text { hyphae not } \\
\text { seen }\end{array}$ & Negative & - & Positive \\
\hline 6. & 19 & $M$ & Fever, cough & $\begin{array}{l}\text { Intravenous } \\
\text { drug abuse }\end{array}$ & TB & 174 & $\begin{array}{l}\text { Fungal } \\
\text { hyphae not } \\
\text { seen }\end{array}$ & Negative & - & Positive \\
\hline 7. & 18 & $\mathrm{M}$ & Fever. Cough & $\begin{array}{c}\text { Heterosexual } \\
\text { contact }\end{array}$ & TB & 317 & $\begin{array}{l}\text { Fungal } \\
\text { hyphae } \\
\text { seen }\end{array}$ & Positive & $\begin{array}{l}\text { Aspergillus } \\
\text { flavus }\end{array}$ & Positive \\
\hline 8. & 42 & M & Fever, cough & $\begin{array}{c}\text { Heterosexual } \\
\text { contact }\end{array}$ & - & 54 & $\begin{array}{l}\text { Fungal } \\
\text { hyphae not } \\
\text { seen }\end{array}$ & Negative & - & Positive \\
\hline 9. & 26 & $M$ & $\begin{array}{l}\text { Fever, } \\
\text { dyspnea }\end{array}$ & $\begin{array}{c}\text { Blood } \\
\text { transfusion }\end{array}$ & TB & 120 & $\begin{array}{c}\text { Fungal } \\
\text { hyphae not } \\
\text { seen }\end{array}$ & Negative & - & Positive \\
\hline 10. & 32 & $M$ & Fever, cough & $\begin{array}{c}\text { Heterosexual } \\
\text { contact }\end{array}$ & $-^{-}$ & 83 & $\begin{array}{l}\text { Fungal } \\
\text { hyphae } \\
\text { seen }\end{array}$ & Positive & $\begin{array}{l}\text { Aspergillus } \\
\text { niger }\end{array}$ & Positive \\
\hline
\end{tabular}

*HIV, Human immunodeficiency virus; †AIDS, Acquired immunodeficiency syndrome; $\not$ IPA, Invasive pulmonary aspergillosis; $\$ M$, Male; ||TB, Tuberculosis; **PCP, Pneumocystis carinii pneumonia

\section{Discussion}

The ever increasing number of immunosuppressed conditions has expanded the spectrum of patients at risk of developing invasive fungal diseases such as IPA. [20, ${ }^{21]}$ Typically, a definite diagnosis is rarely established and diagnosis frequently relies on a combination of clinical features and radiological abnormalities in a patient with high-risk predisposing conditions. While an accurate diagnosis relies on histopathology, a lung biopsy is seldom performed and even in cases where it is feasible the diagnostic accuracy rarely exceeds $80 \%$, more so in event of false negative results due to failure of the invasive method to reach the area infected by Aspergillus species. [22, 23] Additionally, while Aspergillus hyphae can be readily differentiated from the wide, pauciseptate hyphae of mucoraceous moulds, distinguishing them from the narrow, septate hyphae of Fusarium and Scedosporium is often not possible. ${ }^{[1]}$ Culture is time consuming and may delay the diagnosis of IPA. Furthermore, its clinical utility is limited by low sensitivity. ${ }^{[24]}$ Poor sensitivity of mycological cultures for diagnosis of IPA is also evident from our study.

Biomarkers such as serum galactomannan provide a potential adjunct in the diagnosis of IPA. In 2002, galactomannan antigen detection was included as an indirect evidence of a probable invasive fungal disease and has been retained as such in the definition of mycological criteria as described by the updated EORTC/ MSG guidelines published in 2008. ${ }^{[10,25]}$ The sensitivity and specificity of serum galactomannan assay has been reported to vary across different studies. In a meta-analysis by Pffeifer, including pediatric as well as adult population, the sensitivity has been reported to vary between $38-100 \%$ and the specificity between $17-100 \% .{ }^{[4]}$ As postulated by some researchers, serum galactomannan is rapidly cleared by circulating neutrophils in non-neutropenic patients and generally less angioinvasive forms of Aspergillus infection are seen in them that further restricts the access of galactomannan to the blood stream. ${ }^{[26,27,28]}$ Contrary to the expected, we report a high sensitivity of $90 \%$ for serum galactomannan assay in non-neutropenic HIV reactive patients with a probable IPA.

In addition, we found serum galactomannan assay to exhibit $100 \%$ specificity in HIV reactive patients with a probable IPA. Previous published peer-reviewed studies report false positive galactomannan results as high as $5.7 \%$ to $14 \%$ with serum samples. ${ }^{[5,29]}$ Administration of antibiotic preparations containing piperacillin-tazobactam; amoxycillin-clavulanate parenteral preparations; administration of cyclophosphamide; consumption 
of milk preparations containing high concentrations of galactomannan; intravenous administration of the electrolyte solution, Plasmalyte ${ }^{\circledR}$; cross reactions with other moulds such as Pencillium species, Paecilomyces, Fusarium, zygomycetes, Trychophyton and dematiaceous fungi such as Alternaria species that contain cross-reacting epitopes; cross-reactions with antigens from Cryptococcus neoformans and Geotrichum capitatum; and presence of bacteremia, are among the many reasons cited for false reactivity with adult sera. [30, 31, 32, 33, 34, 35, 36, 37] However, serial sampling has been stated to improve the accuracy of serum galactomannan as a screening tool and as per one of the studies, false positivity rates declined from $54.2 \%$ to $11.2 \%$ if positivity for at least two samples was taken as a defining criterion. ${ }^{[18]}$ The authors of the present study have also followed the same criterion and thus false reactivity seems less likely.

In our study, CT scans were only performed in patients with a probable invasive pulmonary aspergillosis and hallmark imaging findings were noted in only one of them. While the performance and utility of CT scans in diagnosis of IPA is beyond the scope of this study, we would surely like to emphasize upon the risk of radiation exposure associated with frequent CT scans and the difficulty encountered in moving unwell patients from protective isolation for this purpose. On the other hand, blood for serum galactomannan assay can be drawn more often and the test can be repeated at frequent intervals. Thus, biomarker assays such as serum galactomannan test have provided an alternative diagnostic approach to accomplish an early and specific diagnosis of IPA.

Our study is fraught with certain limitations. The number of patients included in the analysis was relatively small. Secondly, due to ethical concerns, lung biopsy for a histomorphological demonstration of invasive disease was not performed. As a consequence, galactomannan assay test results could not be verified by the invasive reference test and the true performance characteristics of Platelia ${ }^{\mathrm{TM}}$ Aspergillus EIA could not be determined. Thirdly, thoracic CT scans which are clearly more sensitive than plain chest radiographs, were not performed in all patients. Finally, as with few other studies, a possibility of incorporation bias cannot be ruled out. ${ }^{[38]}$ We have included a positive serum galactomannan result as a microbiological criterion to define probable IPA, and by doing so, we have included the test under evaluation in the reference standard. This accounts for the higher than usual sensitivity and specificity values of serum galactomannan assay in our study. We suggest that larger, well designed, prospective and multi-center studies are undertaken to assess the analytical performance and evaluate the utility of serum galactomannan antigen for diagnosis of IPA in non-neutropenic patient populations such as HIV reactive patients.

We would also like to discuss upon the utility of EORTC/ MSG criteria for diagnosis of IPA in our study group. These definitions were primarily devised for patients with malignant diseases and allogeneic hematopoietic stem cell transplant recipients. ${ }^{[10,38]}$ Their application to non-cancer populations is questionable in view of lack of conclusive evidences to substantiate their utility. Furthermore, the authors of the present study have employed the original EORTC/MSG definitions and not the revised criteria, for categorizing IPA cases into probable and possible. This is because fever, which was included as a host factor in the original definition, was removed in the subsequent revision due to its non-specificity for invasive fungal disease and the plausible reason by consensus group that presence of fever represents a clinical manifestation and not host factor. Thus, while inherited severe immunodeficiency disorders were included in the revised host criteria, febrile illness in an AIDS patient was no longer retained in the definition and therefore the revised EORTC/MSG guidelines could not be applied to our study group. ${ }^{[10,25]}$ Also, the concept of two levels-major and minor evidences, that was originally proposed for the definition of clinical criterion, was abandoned in the revised guidelines, and a diagnosis of probable IPA now relied on documentation of at least one of the three highly specific radiological criteria (dense, well-circumscribed lesions(s) with or without halo sign, air crescent sign or cavity). ${ }^{[10,25]}$ Thus, while the revised EORTC/MSG definitions are more specific, they are also definitely more restrictive and it is quite possible that in the wake of highly specific but less sensitive eligibility criteria the clinical significance of IPA may be underestimated and awareness and consciousness of this entity would soon be lost. Thus, clinicians should keep in mind that these criteria are just meant to stratify the certainty and risk of invasive pulmonary aspergillosis, and to serve as a clinical and epidemiological research guide, and every patient need not fulfill these criteria in order to warrant treatment for IPA.

\section{Conclusion}

To summarize, in the era of diagnostic deficiencies of conventional modalities, serum galactomannan antigen offers a key approach to provide a supportive evidence of IPA. Our study has shown that the assay has a high sensitivity and specificity. However, positive findings need to be cautiously interpreted in the context of clinical and radiological evidences. All positive results should be validated by repeat testing and causes of false positive reactivity such as galactomannan contamination and crossreactive mycoses be ruled out. 


\section{Funding}

This study was funded by the Indian Council of Medical Research

\section{References}

1. Patterson TF. Advances and challenges in management of invasive mycoses. Lancet 2005; 366: 1013-25.

2. Min Z, Baddley JW, Rodriguez JM, Moser SA, Patel M. Cross-reactivity of Aspergillus galactomannan in an HIVinfected patient with histoplasmosis. Med Mycol Case Rep 2012; 1: 119-22.

3. Holding KJ, Dworkin MS, Wan PC, et al. Aspergillosis among people infected with human immunodeficiency virus: incidence and survival. Adult and Adolescent Spectrum of HIV Disease Project. Clin Infect Dis 2000; 31: 1253-7.

4. Pfeiffer CD, Fine JP, Safdar N. Diagnosis of invasive aspergillosis using a galactomannan assay: a meta-analysis. Clin Infect Dis 2006; 42:1417-27.

5. Klont RR, Mennink-Kersten MA, Verweij PE. Utility of Aspergillus antigen detection in specimens other than serum specimens. Clin Infect Dis 2004; 39: 1467-74.

6. Bruno C, Minniti S, Vassanelli A, Pozzi-Mucelli R. Comparison of $\mathrm{CT}$ features of Aspergillus and bacterial pneumonia in severely neutropenic patients. J Thorac Imaging 2007; 22:160-5.

7. Li XS, Zhu HX, Fan HX, Zhu L, Wang HX, Song YL. Pulmonary fungal infections after bone marrow transplantation: the value of high-resolution computed tomography in predicting their etiology. Chin Med J (Engl) 2011; 124:3249-54.

8. Walsh TJ, Anaissie EJ, Denning DW, et al; Infectious Diseases Society of America. Treatment of aspergillosis: clinical practice guidelines of the Infectious Diseases Society of America. Clin Infect Dis 2008; 46:327-60.

9. Hope WW, Walsh TJ, Denning DW. The invasive and saprophytic syndromes due to Aspergillus spp. Med Mycol 2005; 43 Suppl 1:S207-38.

10. Ascioglu S, Rex JH, de Pauw B, et al; Invasive Fungal Infections Cooperative Group of the European Organization for Research and Treatment of Cancer; Mycoses Study Group of the National Institute of Allergy and Infectious Diseases. Defining opportunistic invasive fungal infections in immunocompromised patients with cancer and hematopoietic stem cell transplants: an international consensus. Clin Infect Dis 2002; 34: 7-14.

11. Barton RC. Laboratory diagnosis of invasive aspergillosis: from diagnosis to prediction of outcome. Scientifica (Cairo) 2013; 2013:459405.

12. Steinbach WJ, Addison RM, McLaughlin L, et al. Prospective Aspergillus galactomannan antigen testing in pediatric hematopoietic stem cell transplant recipients. Pediatr Infect Dis J 2007; 26:558-64.

13. Patterson TF. Clinical utility and development of biomarkers in invasive aspergillosis. Trans Am Clin Climatol Assoc 2011; 122: 174-83.
14. Donnelly JP. Polymerase chain reaction for diagnosing invasive aspergillosis: getting closer but still a ways to go. Clin Infect Dis 2006; 42:487-9.

15. Hope WW, Kruhlak MJ, Lyman CA, et al. Pathogenesis of Aspergillus fumigatus and the kinetics of galactomannan in an in vitro model of early invasive pulmonary aspergillosis: implications for antifungal therapy. J Infect Dis 2007; 195:455-66.

16. Mennink-Kersten MA, Donnelly JP, Verweij PE. Detection of circulating galactomannan for the diagnosis and management of invasive aspergillosis. Lancet Infect Dis 2004; 4:349-57.

17. Khanna S, Oberoi J K, Datta S, Aggarwal S, Wattal C. Variables affecting the performance of galactomannan assay in high-risk patients at a tertiary care centre in India. Indian J Med Microbiol 2013;31:34-9

18. Ghosh I, Raina V, Kumar L, Sharma A, Bakhshi S, Iqbal S. Serum galactomannan assay for diagnosis of probable invasive Aspergillosis in acute leukemia and hematopoietic stem cell transplantation. Indian J Med Paediatr Oncol 2013; 34:74-9.

19. Moore GS, Jaciow DM (1979) Mycology for the Clinical Laboratory. Reston, VA: Prentice-Hall, USA.

20. Guinea J, Torres-Narbona M, Gijón P, et al. Pulmonary aspergillosis in patients with chronic obstructive pulmonary disease: incidence, risk factors, and outcome. Clin Microbiol Infect 2010; 16: 870-7.

21. Meersseman W, Vandecasteele SJ, Wilmer A, Verbeken E, Peetermans WE, Van Wijngaerden E. Invasive aspergillosis in critically ill patients without malignancy. Am J Respir Crit Care Med 2004; 170: 621-5.

22. Guarner J, Brandt ME. Histopathologic diagnosis of fungal infections in the 21st century. Clin Microbiol Rev 2011; 24: 247-80.

23. Lopes da Silva R, Ribeiro P, Abreu N, et al. Early diagnosis of invasive aspergillosis in neutropenic patients. Comparison between serum galactomannan and polymerase chain reaction Clinical Medicine Insights: Oncology 2010; 4:81-8.

24. Tarrand JJ, Lichterfeld M, Warraich I, et al. Diagnosis of invasive septate mold infections. A correlation of microbiological culture and histologic or cytologic examination. Am J Clin Pathol 2003; 119:854-8.

25. De Pauw B, Walsh TJ, Donnelly JP, et al; European Organization for Research and Treatment of Cancer/Invasive Fungal Infections Cooperative Group; National Institute of Allergy and Infectious Diseases Mycoses Study Group (EORTC/MSG) Consensus Group. Revised definitions of invasive fungal disease from the European Organization for Research and Treatment of Cancer/Invasive Fungal Infections Cooperative Group and the National Institute of Allergy and Infectious Diseases Mycoses Study Group (EORTC/MSG) Consensus Group. Clin Infect Dis 2008; 46:1813-21. 
26. Guinea J, Jensen J, Peláez $\mathrm{T}$, et al. Value of a single galactomannan determination (Platelia) for the diagnosis of invasive aspergillosis in non-hematological patients with clinical isolation of Aspergillus spp. Med Mycol 2008; 46:575-9.

27. Husain S, Paterson DL, Studer SM, et al. Aspergillus galactomannan antigen in the bronchoalveolar lavage fluid for the diagnosis of invasive aspergillosis in lung transplant recipients. Transplantation 2007; 83:1330-6.

28. Meersseman W, Lagrou K, Maertens J, Van Wijngaerden E. Invasive aspergillosis in the intensive care unit. Clin Infect Dis 2007; 45:205-16.

29. Maertens J, Verhaegen J, Demuynck H, et al. Autopsycontrolled prospective evaluation of serial screening for circulating galactomannan by a sandwich enzyme-linked immunosorbent assay for hematological patients at risk for invasive Aspergillosis. J Clin Microbiol 1999; 37:3223-8.

30. Aubry A, Porcher R, Bottero J, et al. Occurrence and kinetics of false-positive Aspergillus galactomannan test results following treatment with beta-lactam antibiotics in patients with hematological disorders. J Clin Microbiol 2006; 44:389-94.

31. Maertens J, Theunissen K, Lodewyck T, Lagrou K, Van Eldere J. Advances in the serological diagnosis of invasive Aspergillus infections in patients with haematological disorders. Mycoses 2007; 50 Suppl 1:2-17.
32. Viscoli C, Machetti M, Cappellano P, et al. False-positive galactomannan platelia Aspergillus test results for patients receiving piperacillin-tazobactam. Clin Infect Dis 2004; 38:913-6.

33. Racil Z, Kocmanova I, Lengerova M, Winterova J, Mayer J. Intravenous PLASMA-LYTE as a major cause of falsepositive results of platelia Aspergillus test for galactomannan detection in serum. J Clin Microbiol 2007; 45:3141-2.

34. Mikulska M, Furfaro E, Del Bono V, et al. Galactomannan testing might be useful for early diagnosis of fusariosis. Diagn Microbiol Infect Dis 2012; 72:367-9.

35. Huang YT, Hung CC, Hsueh PR. Aspergillus galactomannan antigenemia in penicilliosis marneffei. AIDS 2007; 21:1990-1.

36. Dalle F, Charles PE, Blanc K, et al. Cryptococcus neoformans Galactoxylomannan contains an epitope(s) that is crossreactive with Aspergillus Galactomannan. J Clin Microbiol 2005; 43:2929-31.

37. Bonini A, Capatti C, Parmeggiani M, et al. Galactomannan detection in Geotrichum capitatum invasive infections: report of 2 new cases and review of diagnostic options. Diagn Microbiol Infect Dis 2008; 62:450-2.

38. He H, Ding L, Sun B, Li F, Zhan Q. Role of galactomannan determinations in bronchoalveolar lavage fluid samples from critically ill patients with chronic obstructive pulmonary disease for the diagnosis of invasive pulmonary aspergillosis: a prospective study. Crit Care 2012; 16:R138.

*Corresponding author:

Dr. Ravinder Kaur, Director Professor, Department of Microbiology, Lady Hardinge Medical College and Associated Kalawati Saran Children Hospital, New Delhi-110001, India

Phone: +919811809868

Email: rkaur.mamc@gmail.com

Date of Submission : 27.03.2017

Financial or other Competing Interests: None. 